\title{
Safe and therapeutic care: balancing the risk of opioid prescriptions while effectively treating acute pain
}

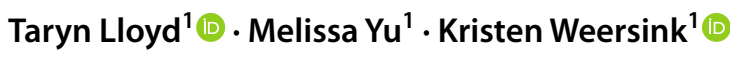

Received: 10 March 2021 / Accepted: 23 March 2021

(C) The Author(s), under exclusive licence to Canadian Association of Emergency Physicians (CAEP)/ Association Canadienne de Médecine d'Urgence (ACMU) 2021

Keywords Opioids $\cdot$ Harm reduction $\cdot$ Acute pain

Emergency medicine providers are frequently caught in the tension between providing effective analgesia for patients and minimizing harms associated with opioid medications. From the front lines of Canada's devastating opioid epidemic, McKinney, Kisilewicz, and Stiell have highlighted how current Canadian emergency physicians are balancing the risk of opioid prescriptions. Caught in the tension, most providers report using gestalt [1] as an imperfect method of assessing the risk of developing harms associated with opioid prescriptions. While we agree with McKinney et al. that the development of an Emergency Department (ED) opioid risk assessment tool is important, we want to emphasize that all patients presenting to the ED with acute pain deserve to have their pain adequately treated and risks minimized by using 'universal precautions'. In this article, we use the term acute pain to refer to self-limited pain provoked by a specific illness or injury, which should be distinguished from chronic or functional pain, both of which are best treated with nonopioid analgesics outside of the ED. How can the individual emergency provider effectively treat pain while minimizing the risk of harms associated with opioid medications?

The use of opioids for acute pain should be a part of a regimen that includes multimodal analgesia (i.e. NSAIDs, acetaminophen, ketamine, local anesthetics) and non-pharmacologic strategies. When this is done, it has been demonstrated by Daoust et al. that most patients effectively optimize non-opioid medications before using opioids, receive pain relief from opioid medications and stop opioid medications within 14-days [2]. Although encouraged by their results, we caution against the authors' recommendation to

Taryn Lloyd

Taryn.Lloyd@unityhealth.to

1 St Michael's Hospital, Unity Health Toronto, 30 Bond St, Toronto, ON, Canada avoid opioids all together if patients have risk factors for opioid use disorder and we're disappointed that they excluded this population entirely in this study. Most emergency physicians are using their gestalt to assess the potential risk of harm with opioid prescriptions [1], a problematic practice sure to introduce implicit bias. To then deny effective opioid analgesic is only further stigmatization of patients in need of care.

Counterintuitively, undertreated pain, particularly in a patient population at risk of aberrant use or opioid use disorder, may cause a myriad of consequences including the development of chronic pain, increased illicit drug use, relapse of illicit drug use [3], and further mistrust of the health care system that discourages individuals from seeking help or treatment when it is needed. Further, there is a solid biochemical explanation to support that patients with opioid use disorder require opioid analgesia in the face of acute pain. First, patients with opioid use disorder likely have developed a high opioid tolerance, whereby higher doses of short-acting opioids are required to obtain the same level of pain relief as those without [3]. Higher doses of opioid analgesia may also be required for patients on opioid agonist therapy, such as buprenorphine and methadone [3]. It is important to remember that opioid agonist therapy is not used for treating pain and these medications will not provide any pain relief on their own. Buprenorphine in particular has a high affinity for opioid receptors, outcompeting many other opioids used in the ED. Higher opioid doses are often needed to overcome this high affinity. Finding the effective dose of a short-acting opioid while a patient is in the monitored setting of an ED can help to guide safe outpatient prescription doses. In addition, patients with opioid use disorder may be experiencing a degree of opioid withdrawal that can be masked as increased pain or a heightened stress 
response [3]. Lastly, chronic use of any opioid can lead to opioid-induced hyperalgesia [3].

Safe and therapeutic care of all patients with acute pain involves the implementation of 'universal precautions'. Tailoring prescriptions to align with the expected course and duration of the acute pain episode is recommended. Interestingly, for acute pain, patients often consume less than the total number of tablets prescribed. Daoust et al. demonstrated that the median number of opioid tablets prescribed was 30 [2], whereas patients actually consume a median of less than 10 opioid tablets [4]. We recommend a short course of opioids from the ED with re-assessment by primary outpatient providers if additional doses are needed. Furthermore, long-acting, controlled-release opioids and combination non-opioid/opioid analgesics should be avoided for the treatment of acute pain. Combination analgesics limit the ability to optimize non-opioid medications that may otherwise be sufficient. Opioid prescriptions of higher daily doses, longer duration, and long-acting opioid formulations are associated with a higher risk of overdose and long-term use [5]. For example, in Ontario it has been found that prescription durations greater than 5 days increased the risk of opioid overdose by 27-80\% [5]. Moreover, all it takes is 5 days of continued opioid use for some patients to develop physical dependence. Prescribing in short dispensing intervals (i.e., every 1-3 days) is another strategy to reduce potential aberrant use of prescription opioids.

Harm reduction counselling is paramount for all patients that are prescribed opioids. The risk of opioid toxicity (respiratory depression, accidental overdose, and death) should be reviewed, including the increased risk for those who take sedatives (benzodiazepines, gabapentin, antipsychotics) or consume alcohol concurrently. Prescription for a naloxone kit, education of safe storage, disposal of unused medications, and avoidance of driving while taking opioids should be advised for all patients. Close follow-up with a primary care provider should also be arranged. Communication back to primary care physicians and pharmacies can help mitigate harm regardless of individual risk factors. If specific concerns arise of aberrant opioid use or dependency, concurrent treatment of acute pain and referral to an addiction medicine specialist should be pursued in a supportive fashion for patient-centered care.

With attention and care, emergency physicians can provide safe and effective pain relief with opioids to all patients when clinically indicated. We commend the authors McKinney et al. for calling for improved risk assessment tools and system-level initiatives for safe opioid prescribing [1], and Daoust et al. for highlighting the ongoing role of opioids in acute pain management from the ED setting [2]. Patients and ED providers would benefit from ongoing improvements to validated tools to assess opioid risk and identify at-risk patients early for increased support such as social work, referral to addiction medicine, and specific tailored prescribing.

\section{Declarations}

Conflict of interest The authors declare that there are no conflicts of interest.

\section{References}

1. McKinney M, Kisilewicz M, Stiell IG. Emergency physician risk assessment practices prior to prescribing opioids. Can J Emerg Med Care. 2021. https://doi.org/10.1007/s43678-020-00066-8.

2. Daoust R, Paquet J, Cournoyer A, et al. Opioid and nonopioid pain relief after an emergency department acute pain visit. Can J Emerg Med Care. 2021. https://doi.org/10.1007/ s43678-020-00041-3.

3. Alford DP, Compton P, Samet JH. Acute pain management for patients receiving maintenance methadone or buprenorphine therapy. Ann Intern Med. 2006;144(2):127-34.

4. Daoust R, Paquet J, Cournoyer A, et al. Quantity of opioids consumed following an emergency department visit for acute pain: a Canadian prospective cohort study. BMJ Open. 2018;8(9):e022649. https://doi.org/10.1136/bmjop en-2018-022649.

5. Gomes T, Campbell T, Tadrous M, Mamdani MM, Paterson JM, Juurlink DN. Initial opioid prescription patterns and the risk of ongoing use and adverse outcomes. Pharmacoepidem Dr S. 2021;30(3):379-89. https://doi.org/10.1002/pds.5180. 